\title{
PROJECTIONS, THE WEIGHTED BERGMAN SPACES, AND THE BLOCH SPACE
}

\author{
BOO RIM CHOE
}

(Communicated by Clifford J. Earle, Jr.)

\begin{abstract}
It has been known that there is a family of projections $P_{S}$ of the Lebesgue spaces onto the Bergman spaces on the unit ball of $\mathbb{C}^{n}(n \geq 1)$. The corresponding result for the weighted Bergman spaces $A_{\alpha}^{p}$ is obtained. As applications a solution of Gleason's problem at the origin for $A_{\alpha}^{p}$ and a characterization of $A_{\alpha}^{p}$ in terms of partial derivatives are indicated without proof. Also the natural limiting case is found: $P_{s} L^{\infty}=\mathfrak{B}$, the Bloch space, and $P_{s} C_{0}=\mathfrak{B}_{0}$, the little Bloch space. Moreover, simple bounded linear operators $L_{s}: \mathfrak{B} \rightarrow L^{\infty}$, with $L_{s}\left(\mathfrak{B}_{0}\right) \subset C_{0}$, are found so that $P_{s} \circ L_{s}$ is the identity on $\mathfrak{B}$. As an application the dualities $\mathfrak{B}=\left(A_{\alpha}^{1}\right)^{*}$ and $\mathfrak{B}_{0}^{*}=A_{\alpha}^{1}$ are established under each of pairings suggested by projections $P_{S}$.
\end{abstract}

\section{INTRODUCTION}

The results of the present paper extend and complement those of ForelliRudin [6], Kolaski [7], and Zhu [13].

Let $\mathbb{C}^{n}$ denote the $n$-dimensional complex space of all ordered $n$-tuples $z=$ $\left(z_{1}, \ldots, z_{n}\right)$ of complex numbers with inner product $z \cdot \bar{w}=z_{1} \bar{w}_{1}+\cdots+z_{n} \bar{w}_{n}$. The unit ball $B$ of $\mathbb{C}^{n}$ is then the set of all $z \in \mathbb{C}^{n}$ with $|z|=(z \cdot \bar{z})^{1 / 2}<1$. We shall let $H(B)$ denote the class of holomorphic functions on $B$ and let $A(B)=H(B) \cap C(\bar{B})$.

The Bloch space $\mathfrak{B}$ is the space of all $g \in H(B)$ for which

$$
\|g\|_{\mathfrak{B}}=|g(0)|+\sup _{z \in B}\left(1-|z|^{2}\right)|\nabla g(z)|<\infty
$$

where $\nabla g=\left(D_{1} g, \ldots, D_{n} g\right), D_{j}=\partial / \partial z_{j}(j=1, \ldots, n)$, is the complex gradient of $g$. The norm \|\|$_{\mathfrak{B}}$ turns the space $\mathfrak{B}$ into a Banach space. For a long list of equivalent definitions of the Bloch space, see [8] and [11], where the settings are more general, and [2], where the setting is the unit disc. The little Bloch space $\mathfrak{B}_{0}$ is the closed subspace of $\mathfrak{B}$, consisting of functions $g$ for which the additional condition $\left(1-|z|^{2}\right)|\nabla g(z)|=o(1)$ holds. Let $d V$ denote the normalized volume measure on $B$ and define a measure $d V_{\alpha}$

Received by the editors February 9, 1989.

1980 Mathematics Subject Classification (1985 Revision). Primary 32A25; Secondary 47B38.

Key words and phrases. Projections, weighted Bergman spaces, Bloch space. 
( $\alpha>-1$ is fixed throughout the paper unless otherwise specified) on $B$ by $d V_{\alpha}(z)=\left(1-|z|^{2}\right)^{\alpha} d V(z)$. The weighted Bergman space $A_{\alpha}^{p} \quad(1 \leq p<\infty)$ is then the closed subspace $L^{p}\left(V_{\alpha}\right) \cap H(B)$ of $L^{p}\left(V_{\alpha}\right)$. We will use the fact that $A(B)$ is dense in both $\mathfrak{B}_{0}$ and $A_{\alpha}^{p}$.

Corresponding to a complex number

$$
s=\sigma+i t \quad(\sigma>-1,-\infty<t<\infty),
$$

we define a kernel

$$
K_{s}(z, w)=\frac{\left(1-|w|^{2}\right)^{s}}{(1-z \cdot \bar{w})^{n+1+s}} \quad(z \in B, w \in B)
$$

and an integral operator

$$
P_{s} f(z)=\lambda_{s} \int_{B} K_{s}(z, w) f(w) d V(w) \quad(z \in B)
$$

where $\lambda_{s}=\Gamma(n+s+1) / \Gamma(n+1) \Gamma(s+1)$ and the complex powers that occur in the kernel $K_{s}$ are understood to be principal branches.

Theorem 1. For $1 \leq p<\infty, P_{s}$ is a bounded operator on $L^{p}\left(V_{\alpha}\right)$ if and only if $p(1+\sigma)>1+\alpha$. If $p(1+\sigma)>1+\alpha$, then $P_{s} f=f$ and $P_{s} \bar{f}=\overline{f(0)}$ for every $f \in A_{\alpha}^{p}$.

Shields and Williams [10] proved Theorem 1 for $s>0, p=1$ in the unweighted case $(\alpha=0)$ on the unit disc. Then Forelli and Rudin [6] proved the unweighted case of Theorem 1, which appears in [9] as Theorem 7.1.4. On the other hand, Kolaski [7] obtained Theorem 1 in the case $\sigma=\alpha$. As indicated in [6], [7] and [9], Theorem 1 does not extend to the case $p=\infty$. There is, however, a natural limiting case, which was not emphasized by earlier authors:

Theorem 2. For every $s, P_{s}$ is a bounded operator from $L^{\infty}(B)$ onto $\mathfrak{B}$. Moreover, $P_{s} C(\bar{B})=P_{s} C_{0}(B)=\mathfrak{B}_{0}$.

Here $C_{0}(B)=\{f \in C(\bar{B}):|f(z)| \rightarrow 0$ as $|z| \rightarrow 1\}$. In fact we will construct simple bounded linear operators $L_{s}: \mathfrak{B} \rightarrow L^{\infty}(B)$, with $L_{s}\left(\mathfrak{B}_{0}\right) \subset C_{0}(B)$, so that $P_{s} \circ L_{s}$ is the identity on $\mathfrak{B}$ (see Corollary 13). This also establishes the dualities $\mathfrak{B}=\left(A_{\alpha}^{1}\right)^{*}$ and $A_{\alpha}^{1}=\mathfrak{B}_{0}^{*}$ (superscript * denotes the dual space) under each of pairings suggested by $P_{s}$ (see Theorems 15 and 16). In a recent paper [14] Zhu has solved a generalized Gleason problem at the origin for the unweighted Bergman spaces and obtained a characterization of those spaces in terms of partial derivatives of their elements. The main ingredients in Zhu's work are the unweighted cases of Theorem 1, Proposition 6, and its consequence Corollary 7 . We therefore obtain the corresponding weighted versions, Theorems 3 and 4 , for free: one imitates, line by line, proofs of Theorems $4,5,11$, and 21 of [14], and thus details are left to the readers. Here we use the convenient multi-index notation: for ordered $n$-tuples $\beta=\left(\beta_{1}, \ldots, \beta_{n}\right)$ of nonnegative integers, we write $|\beta|=\beta_{1}+\cdots+\beta_{n}, z^{\beta}=z_{1}^{\beta_{1}} \cdots z_{n}^{\beta_{n}}$, and $D^{\beta}=D_{1}^{\beta_{1}} \cdots D_{n}^{\beta_{n}}$. 
Theorem 3. For each multi-index $\beta$ there exists a linear operator $G_{\beta}$ bounded on $A_{\alpha}^{p}(1 \leq p<\infty)$ such that if $f \in A_{\alpha}^{p}$ and all its partial derivatives of order $\leq m-1$ are zero at 0 for some positive integer $m$, then $f(z)=\sum z^{\beta} G_{\beta} f(z)$ on $B$, where the sum is taken over all $\beta$ with $|\beta|=m$.

Theorem 4. Let $1 \leq p<\infty$ and $m$ be a positive integer. Then a function $f \in H(B)$ belongs to $A_{\alpha}^{p}$ if and only if $D^{\beta} f \in A_{p m+\alpha}^{p}$ for every multi-index $\beta$ with $|\beta|=m$. Moreover, the norm of $f \in A_{\alpha}^{p}$ is equivalent to

$$
\sum_{|\beta| \leq m-1}\left|D^{\beta} f(0)\right|+\sum_{|\beta|=m}\left\|D^{\beta} f\right\|_{A_{p m+a}^{p}} .
$$

The inclusion $\mathfrak{B} \subset A_{\alpha}^{p}$ is clear from Theorem 4 (with $m=1$ ).

\section{THEOREM 1}

For $\delta>-1$ and $c$ real, define

$$
J_{c, \delta}(z)=\int_{B} \frac{\left(1-|w|^{2}\right)^{\delta}}{|1-z \cdot \bar{w}|^{n+1+\delta+c}} d V(w) \quad(z \in B) .
$$

Note that $J_{c, \delta}(z)$ is a function of $|z|$. A proof of the following lemma can be found in Proposition 1.4.10 of [9]. In this paper, only the "big oh" consequence of the lemma is important.

Lemma 5. If $c>0$, then $\left(1-|z|^{2}\right)^{c} J_{c, \delta}(z)$ has a positive finite limit as $|z| \rightarrow 1$.

Note. In the above lemma one can conclude a bit stronger result asserting that $\left(1-|z|^{2}\right)^{c} J_{c, \delta}(z)=J_{-c, \delta}(z)$ (see Appendix) on $B$ for every real $c$ and thus $\left(1-|z|^{2}\right)^{c} J_{c, \delta}(z)$ increases to $J_{-c, \delta}(\xi) \quad(\xi \in \partial B)$ as $|z|$ increases to 1 for $c>0$.

We first prove a stronger version of the first part of Theorem 1 . We define

$$
T_{\sigma} f(z)=\int_{B} \frac{\left(1-|w|^{2}\right)^{\sigma}}{|1-z \cdot \bar{w}|^{n+1+\sigma}} f(w) d V(w) \quad(z \in B) .
$$

Proposition 6. For $1 \leq p<\infty, T_{\sigma}$ is bounded on $L^{p}\left(V_{\alpha}\right)$ if and only if $p(1+$ $\sigma)>1+\alpha$.

Proof. We will consider only the case $1<p<\infty$ : the case $p=1$ can be treated in exactly the same way as in the proof of Theorem 7.1.4 of [9]. The proof that follows is also an easy modification.

Assume that $1<p<\infty$ and put $T_{\sigma}(z, w)=\left(1-|w|^{2}\right)^{\sigma-\alpha}|1-z \cdot \bar{w}|^{-(n+1+\sigma)}$ so that

$$
T_{\sigma} f(z)=\int_{B} T_{\sigma}(z, w) f(w) d V_{\alpha}(w) .
$$


Let $q$ be the conjugate exponent of $p$. If $p(1+\sigma) \leq 1+\alpha$, then $q(\sigma-$ $\alpha)+\alpha \leq-1$ and thus

$$
\int_{B}\left|T_{\sigma}(z, w)\right|^{q} d V_{\alpha}(w)=\infty
$$

for every $z \in B$, and $T_{\sigma} f$ fails to exist for some $f \in L^{p}\left(V_{\alpha}\right)$. If $p(1+\sigma)>$ $1+\alpha$, then $\delta=\sigma-(1+\alpha) p^{-1}>-1, \varepsilon=(1+\alpha) p^{-1}-1>-1$ and $\sigma-\varepsilon>0$. Define $\varphi(z)=\left(1-|z|^{2}\right)^{-(1+\alpha) / p q}$. Then Lemma 5 shows

$$
\int_{B} T_{\sigma}(z, \cdot) \varphi^{q} d V_{\alpha}=\int_{B} \frac{\left(1-|w|^{2}\right)^{\delta}}{|1-z \cdot \bar{w}|^{n+1+\sigma}} d V(w) \leq a^{q}|\varphi(z)|^{q}
$$

and

$$
\int_{B} T_{\sigma}(\cdot, w) \varphi^{p} d V_{\alpha}=\left(1-|w|^{2}\right)^{\sigma-\alpha} \int_{B} \frac{\left(1-|z|^{2}\right)^{\varepsilon}}{|1-z \cdot \bar{w}|^{n+1+\sigma}} d V(z) \leq b^{p}|\varphi(w)|^{p}
$$

for some constants $a$ and $b$ depending only on $n, \sigma, \alpha$, and $p$. Now the same argument as in the proof of Theorem 7.1.4 of [9] (or Lemma 3.1 of [6]) leads from (1) and (2) to

$$
\int_{B}\left|T_{\sigma} f\right|^{p} d V_{\alpha} \leq(a b)^{p} \int_{B}|f|^{p} d V_{\alpha}
$$

for $f \in L^{p}\left(V_{\alpha}\right)$. The proof is complete.

Corollary 7. Let $1<q \leq \infty$. Then the operator $T_{\sigma}^{*}$ defined by

$$
T_{\sigma}^{*} f(z)=\left(1-|z|^{2}\right)^{\sigma-\alpha} \int_{B} \frac{f(w)}{|1-z \cdot \bar{w}|^{n+1+\sigma}} d V_{\alpha}(w)
$$

is bounded on $L^{q}\left(V_{\alpha}\right)$ if and only if $\sigma-\alpha+(1+\alpha) q^{-1}>0$.

Note that if $q=\infty$, then the above corollary reduces to the "big oh" consequence of Lemma 5.

Proof of Theorem 1. The first part of the theorem is contained in Proposition 6 because

$$
e^{-\pi|t| / 2} \leq\left|K_{s}(z, w)\right|\left|K_{\sigma}(z, w)\right|^{-1} \leq e^{\pi|t| / 2}
$$

on $B \times B$. Recall that the theorem is true for $\alpha=0$. Thus the second part of the theorem follows from the fact that $A(B)$ is dense in $A_{\alpha}^{p}$.

The following corollary combined with Corollary 7 is the adjoint version of Theorem 1.

Corollary 8. Let $1<q \leq \infty$ and assume that $\sigma-\alpha+(1+\alpha) q^{-1}>0$. Then $Q$ is a bounded projection of $L^{q}\left(V_{c}\right)$ onto $M X^{q}$ where (recall $\operatorname{Re} s=\sigma$ )

$$
\begin{gathered}
Q f(z)=\lambda_{s}\left(1-|z|^{2}\right)^{s-\alpha} \int_{B} \frac{f(w)}{(1-z \cdot \bar{w})^{n+1+s}} d V_{\alpha}(w), \\
M f(z)=f(z)\left(1-|z|^{2}\right)^{s-\alpha}, \\
X^{q}=\left\{f \in H(B): M f \in L^{q}\left(V_{\alpha}\right)\right\} .
\end{gathered}
$$


Note that $X^{q}=A_{q(\sigma-\alpha)+\alpha}^{q}$ for $q<\infty$. If $q=\infty$, then the above corollary partially generalizes a result of Shields and Williams ([10], Theorem 1 with $\varphi(r)=\left(1-r^{2}\right)^{\sigma-\alpha}, \psi(r)=\left(1-r^{2}\right)^{\alpha}$, and $\left.s=\sigma\right)$ as well as its higher dimensional version ([12], Theorem 3.16).

\section{THEOREM 2}

In the rest of the paper we fix $s$ and assume that $\sigma=\alpha$ : thus $s=\alpha+i t$. We will use the "inner product" notation

$$
\langle h, g\rangle=\int_{B} h \bar{g} d V_{\alpha}
$$

whenever the integral on the right side of the above exists.

Lemma 9. $P_{s}: L^{\infty}(B) \rightarrow \mathfrak{B}$ is a bounded operator. Moreover, $P_{s} C(\bar{B}) \subset \mathfrak{B}_{0}$.

Proof. Let $\psi \in L^{\infty}(B)$ be given. Write $g=P_{s} \psi$ and differentiate under the integral sign to obtain

$$
D_{j} g(z)=\lambda_{s}(n+1+s) \int_{B} \frac{\left(1-|w|^{2}\right)^{s}}{(1-z \cdot \bar{w})^{n+2+s}} \bar{w}_{j} \psi(w) d V(w) \quad(z \in B)
$$

for $j=1, \ldots, n$. It follows from Lemma 5 that $P_{s}: L^{\infty}(B) \rightarrow \mathfrak{B}$ is bounded.

In the integral of (3), one can replace $\psi(w)$ by $\psi(w)-\psi(z)$, for each fixed $z \in B$, by Theorem 1 without changing the integral. It follows that the integral in the right side of (3) is dominated by some constant (independent of $z$ ) times

$$
\int_{B} \frac{|\psi(z)-\psi(w)|}{|1-z \cdot \bar{w}|^{n+2+\alpha}} d V_{\alpha}(w)
$$

The integral in (4) is easily seen to be $o\left(\left(1-|z|^{2}\right)^{-1}\right)$ by Lemma 5 when $\psi$ is uniformly continuous. Thus $P_{s} C(\bar{B}) \subset \mathfrak{B}_{0}$.

Define $M_{t} f(z)=f(z)\left(1-|z|^{2}\right)^{i t}$. It is then easily verified that $\left\langle P_{\bar{s}} h, M_{t} g\right\rangle=$ $\left\langle h, M_{t} P_{s} g\right\rangle$ whenever an application of Fubini's theorem is justified. In particular, if $h \in A(B)$, this reduces to

$$
\left\langle h, M_{t} g\right\rangle=\left\langle h, M_{t} P_{s} g\right\rangle .
$$

We also define

$$
L_{s} g(z)=(s+1)^{-1}\left(1-|z|^{2}\right)[(n+s+1) g(z)+\mathbf{R} g(z)] \quad(z \in B)
$$

where $\mathbb{R} g(z)=z_{1} D_{1} g(z)+\cdots+z_{n} D_{n} g(z)$ denotes the radial derivative of $g \in H(B)$.

Lemma 10. $L_{s}: \mathfrak{B} \rightarrow L^{\infty}(B)$ is a bounded operator. Moreover, $L_{s} \mathfrak{B}_{0} \subset C_{0}(B)$.

Proof. Since $|\mathbb{R} g| \leq|\nabla g|$ for $g \in H(B)$, it is enough to show that

$$
\left(1-|z|^{2}\right)|g(z)| \leq 2\|g\|_{\mathfrak{B}} \quad(g \in \mathfrak{B})
$$


and

$$
\lim _{|z| \rightarrow 1}\left(1-|z|^{2}\right)|g(z)|=0 \quad\left(g \in \mathfrak{B}_{0}\right) .
$$

It is an easy consequence of the definition of the Bloch space that if $g \in \mathfrak{B}$, then $|g(z)| \leq 2\|g\|_{\mathfrak{B}}\left(1-\log \left(1-|z|^{2}\right)\right)$ on $B$. Thus (6) and (7) follow from the fact that $x(1-\log x) \leq 1$ for $0<x \leq 1$ and $x(1-\log x) \rightarrow 0$ as $x \rightarrow 0$.

Lemma 11. For $h \in A(B), g \in \mathfrak{B}, j=1, \ldots, n$,

$$
(\bar{s}+1)\left\langle z_{j} h, M_{t} g\right\rangle=\left\langle h,\left(1-|z|^{2}\right) M_{t} D_{j} g\right\rangle .
$$

Proof. Since (holomorphic) monomials span a dense subset of $A_{\alpha}^{2}$, we may assume that $h$ is a monomial. Also note that monomials are pairwise orthogonal with respect to any finite rotation-invariant measure on $B$. Thus, by a consideration of the Taylor series expansion of $g$, it suffices to verify (8) for the special case where $h(z)=z^{\beta}$ and $g(z)=z_{j} z^{\beta}$ for some multi-index $\beta$. Namely, it is sufficient to show

(9) $(\bar{s}+1) \int_{B}\left|z^{\beta}\right|^{2}\left|z_{j}\right|^{2}\left(1-|z|^{2}\right)^{-i t} d V_{\alpha}(z)=\left(\beta_{j}+1\right) \int_{B}\left|z^{\beta}\right|^{2}\left(1-|z|^{2}\right)^{1-i t} d V_{\alpha}(z)$

and for every multi-index $\beta=\left(\beta_{1}, \ldots, \beta_{n}\right)$ and for every $j=1, \ldots, n$. Express both sides of (9) in polar coordinates and use the norm formula (see Proposition 1.4 .9 of [9]):

$$
\int_{\partial B}\left|\xi^{\beta}\right|^{2} d S(\xi)=\frac{(n-1) ! \beta_{1} ! \cdots \beta_{n} !}{(n-1+|\beta|) !}
$$

for every multi-index $\beta=\left(\beta_{1}, \ldots, \beta_{n}\right)$ where $d S$ denotes the normalized surface area measure on $\partial B$. The rest of the proof is then a routine calculation and therefore omitted.

Theorem 12. For every $h \in A(B)$ and $g \in \mathfrak{B}$,

$$
\left\langle h, M_{t} g\right\rangle=\left\langle h, M_{t} L_{s} g\right\rangle
$$

and the same is true for every $h \in A_{\alpha}^{1}$ and $g \in A(B)$.

Proof. First assume that $h \in A(B)$ and $g \in \mathfrak{B}$. Then, by (6), $z_{j} g \in \mathfrak{B}$. Thus, by Lemma 11 with $z_{j} g$ in place of $g$, we have

$$
(\bar{s}+1)\left\langle h,\left|z_{j}\right|^{2} M_{t} g\right\rangle=\left\langle h,\left(1-|z|^{2}\right) M_{t}\left(g+z_{j} D_{j} g\right)\right\rangle .
$$

Take the sum, from $j=1$ up to $j=n$, of both sides of (11). Then a little rearrangement of the resulting expression yields (10). Formula (10) remains valid for $h \in A_{\alpha}^{1}$ and $g \in A(B)$ because $A(B)$ is dense in $A_{\alpha}^{1}$.

Corollary 13. $P_{s} \circ L_{s}$ is the identity on $\mathfrak{B}$.

Proof. Let $g \in \mathfrak{B}$. By Lemmas 9 and $10, P_{s} L_{s} g \in \mathfrak{B}$. Now, by first applying Theorem 12 and then (5), we obtain $\left\langle z^{\beta}, M_{t} g\right\rangle=\left\langle z^{\beta}, M_{t} L_{s} g\right\rangle=\left\langle z^{\beta}, M_{t} P_{s} L_{s} g\right\rangle$ 
for every multi-index $\beta$. This implies that $g$ and $P_{s} L_{s} g$ have the same Taylor coefficients. Thus $g=P_{s} L_{s} g$. The proof is complete.

Proof of Theorem 2. The theorem follows immediately from Lemmas 9 and 10 and Corollary 13.

Remark 14. In the case $s=0$ and $n=1,(10)$ takes the simple form

$$
\int_{U} h \bar{g} d A=\int_{U} h(z)(2 \overline{g(z)}+\overline{\mathbb{R} g(z)})\left(1-|z|^{2}\right) d A(z)
$$

where $d A$ denotes the area measure on the unit disc $U$. A similar formula appears in [3, p. 16]:

$$
\int_{U} h \bar{g} d A=\int_{U} z^{-1}(h(z)-h(0)) \overline{g^{\prime}(z)}\left(1-|z|^{2}\right) d A(z)+\pi h(0) \overline{g(0)} .
$$

A little manipulation shows that formulas (12) and (13) are identical. But (12) has the obvious advantage over (13) that it immediately produces the formula

$$
P\left[\left(1-|z|^{2}\right)(2 g+\mathbb{R} g)\right]=g \quad(g \in \mathfrak{B})
$$

where $P$ denotes the Bergman projection on $L^{2}(U)$. Moreover, it can be easily generalized to higher dimensional cases. In fact, Theorem 12 was first proved in this simplest case and then generalized, without knowledge of [3]: the author would like to thank Wade Ramey for drawing his attention to the reference [3]. Explicit (and complicated) formulas like (14) can be found in [3, p. 22] as well. However, they do not appear to be as natural as (14): only the natural factor $\left(1-|z|^{2}\right)$, the radial derivative, and a constant multiple are involved there.

\section{Dualities}

It is probably well known that the dual of $A_{\alpha}^{1}$ is $\mathfrak{B}$ and its predual is $\mathfrak{B}_{0}$ under the pairing $h \times g \rightarrow\langle h, g\rangle$. In the setting of the unit disc these dualities, under the same or a slightly different pairing, in the weighted or unweighted case, can be found in many places, such as [1], [2], [3], [4], [5], and [10]. For the setting of the unit ball, see [12] and [13]. In this section the "same" dualities are established under each of the pairings $h \times g \rightarrow\left\langle h, M_{t} g\right\rangle$. The derivation below is simple and independent of those to be found in the references listed above. The first half of the dualities is almost immediate from Theorem 12:

Theorem 15. Every $g \in \mathfrak{B}$ induces $\Lambda_{g} \in\left(A_{\alpha}^{1}\right)^{*}$ defined by

$$
\Lambda_{g}(h)=\left\langle h, M_{t} g\right\rangle \quad(h \in A(B))
$$

and, conversely, to each $\Lambda \in\left(A_{\alpha}^{1}\right)^{*}$ there corresponds a unique $g \in \mathfrak{B}$ such that $\Lambda=\Lambda_{g}$. Moreover, the norm of $\Lambda_{g}$ is equivalent to the norm of $g \in \mathfrak{B}$.

Proof. By Lemma 10, Theorem 12, and Hölder's inequality, every $g \in \mathfrak{B}$ induces $\Lambda_{g} \in\left(A_{\alpha}^{1}\right)^{*}$ such that $\left\|\Lambda_{g}\right\| \leq\left\|L_{s}\right\|\|g\|_{\mathfrak{B}}$, where $\left\|L_{s}\right\|$ denotes the norm of $L_{s}: \mathfrak{B} \rightarrow L^{\infty}(B)$. 
Now assume that $\Lambda \in\left(A_{\alpha}^{1}\right)^{*}$. The Hahn-Banach theorem then implies the existence of $\psi \in L^{\infty}(B)$ such that $\|\psi\|_{\infty}=\|\Lambda\|$ and $\Lambda(h)=\left\langle h, M_{t} \psi\right\rangle$ for every $h \in A(B)$. In other words, $\Lambda=\Lambda_{g}$ where $g=P_{s} \psi \in \mathfrak{B}$ by (5) and Theorem 2. Note that $\|g\|_{\mathfrak{B}} \leq\left\|P_{s}\right\|\|\psi\|_{\infty}=\left\|P_{s}\right\|\|\Lambda\|$, where $\left\|P_{s}\right\|$ is the norm of $P_{s}: L^{\infty}(B) \rightarrow \mathfrak{B}$. The uniqueness is easily seen as in the proof of Corollary 13. The proof is complete.

The space of finite Borel measures $\mu$ on $\bar{B}$ will be denoted by $M(\bar{B})$ and identified with the dual of $C(\bar{B})$ under the pairing $\mu \times \varphi \rightarrow \mu(\varphi)=\int_{\bar{B}} \bar{\varphi} d \mu$.

Theorem 16. Every $h \in A_{\alpha}^{1}$ induces $\Phi_{h} \in \mathfrak{B}_{0}^{*}$ defined by

$$
\Phi_{h}(g)=\left\langle h, M_{t} g\right\rangle \quad(g \in A(B))
$$

and, conversely, to each $\Phi \in \mathfrak{B}_{0}^{*}$ there corresponds a unique $h \in A_{\alpha}^{1}$ such that $\Phi=\Phi_{h}$. Moreover, the norm of $\Phi_{h}$ is equivalent to the norm of $h \in A_{\alpha}^{1}$.

Proof. As in the proof of Theorem 15, every $h \in A_{\alpha}^{1}$ induces $\Phi_{h} \in \mathfrak{B}_{0}^{*}$ such that $\left\|\Phi_{h}\right\| \leq\left\|L_{s}\right\|\|h\|_{1}$, where $\|h\|_{1}$ denotes the $A_{\alpha}^{1}$ norm of $h$.

Now assume that $\Phi \in \mathfrak{B}_{0}^{*}$. Then $\ell=\Phi \circ P_{s}$ is a continuous linear functional on $C(\bar{B})$ by Theorem 2 and thus there exists a measure $\mu \in M(\bar{B})$ such that $\ell(\varphi)=\mu(\varphi)$ for every $\varphi \in C(\bar{B})$. If $\varphi \in C_{c}(B)$, the subspace of $C(\bar{B})$ consisting of functions supported on compact subsets of $B$, then $P_{s} \varphi \in A(B)$. Thus, by Theorem 1,

$$
\ell(\varphi)=\ell\left(P_{s} \varphi\right)=\int_{\bar{B}} \overline{P_{s} \varphi} d \mu \quad\left(\varphi \in C_{c}(B)\right) .
$$

An application of Fubini's theorem on the right side of (15) is easily justified because $\varphi \in C_{c}(B)$. Thus we have

$$
\ell(\varphi)=\left\langle h, M_{t} \varphi\right\rangle \quad\left(\varphi \in C_{c}(B)\right)
$$

where

$$
h(z)=\bar{\lambda}_{s} \int_{\bar{B}} \frac{d \mu(w)}{(1-z \cdot \bar{w})^{n+1+\bar{s}}} \quad(z \in B) .
$$

Note that $h \in H(B)$. In addition, it follows from (16) that

$$
\int_{r B}|h| d V_{\alpha} \leq\|\Phi\|\left\|P_{s}\right\|
$$

for every $r \in(0,1)$ where $\left\|P_{s}\right\|$ denotes the norm of $P_{s}: C(\bar{B}) \rightarrow \mathfrak{B}_{0}$. Accordingly, $h \in A_{\alpha}^{1}$ and $\|h\|_{1} \leq\|\Phi\|\left\|P_{s}\right\|$ by Fatou's lemma.

Now we prove that $\Phi$ is induced by $h$. Fix $\varphi \in C_{c}(B)$ and put $g=P_{s} \varphi \in$ $A(B)$. Note that if $f \in A(B)$, then $\left\langle f, M_{t} \varphi\right\rangle=\left\langle f, M_{t} L_{s} g\right\rangle$ by (5) and (10). Also $\Phi_{h}(g)=\left\langle h, M_{t} L_{s} g\right\rangle$ by (10). Thus by (16), $\Phi_{h}(g)-\Phi(g)=\langle h-f$, $\left.M_{t}\left(L_{s} g-\varphi\right)\right\rangle$ holds for every $f \in A(B)$. This implies that $\Phi_{h}(g)=\Phi(g)$ by Lemma 10 and Hölder's inequality because $A(B)$ is dense in $A_{\alpha}^{1}$. In summary, $\Phi_{h}=\Phi$ on $P_{s} C_{c}(B)$, which is a dense subset of $\mathfrak{B}_{0}$ by Theorem 2 , because 
$C_{c}(B)$ is uniformly dense in $C_{0}(B)$. Thus $\Phi=\Phi_{h}$. The uniqueness is easily verified as before. The proof is complete.

\section{APPENDIX}

Let $J_{c, \delta}$ denote the function defined at the beginning of the second section. Proposition 17. For $\delta>-1, c$ real, and $z \in B,\left(1-|z|^{2}\right)^{c} J_{c, \delta}(z)=J_{-c, \delta}(z)$. Proof. Let $\operatorname{Aut}(B)$ denote the automorphism group of $B$ consisting of all biholomorphic self-maps of $B$.

Fix $z \in B$. Since $\operatorname{Aut}(B)$ acts transitively on $B$, there exists a function $\varphi \in \operatorname{Aut}(B)$ such that $\varphi(z)=0$. Then (see Theorem 2.2.5 of [9]) we have $\varphi(0)=U z$ for some unitary transformation $U$ on $\mathbb{C}^{n}$. Thus we may assume that $\varphi(0)=z$ as well. We also have the identity

$$
1-\varphi(a) \cdot \overline{\varphi(b)}=\frac{(1-z \cdot \bar{z})(1-a \cdot \bar{b})}{(1-a \cdot \bar{z})(1-z \cdot \bar{b})} \quad(a \in B, b \in B) .
$$

Define a measure $d \tau$ on $B$ by $d \tau(w)=\left(1-|w|^{2}\right)^{-n-1} d V(w)$. Then $d \tau$ is $\operatorname{Aut}(B)$-invariant (see Theorem 2.2.6 of [9]) and therefore we have

$$
\begin{aligned}
J_{c, \delta}(z) & =\int_{B} \frac{\left(1-|\varphi(w)|^{2}\right)^{n+1+\delta}}{|1-\varphi(0) \cdot \overline{\varphi(w)}|^{n+1+\delta+c}} d \tau(w) \\
& =\left(1-|z|^{2}\right)^{-c} \int_{B} \frac{\left(1-|w|^{2}\right)^{n+1+\delta}}{|1-z \cdot \bar{w}|^{n+1+\delta-c}} d \tau(w) \\
& =\left(1-|z|^{2}\right)^{-c} J_{-c, \delta}(z) .
\end{aligned}
$$

In the above the second equality follows from (17). The proof is complete.

\section{REFERENCES}

1. P. Ahern, On the behavior near a torus of functions holomorphic in the ball, Pacific J. Math. 107 (1983), 267-278.

2. J. Anderson, J. Clunie, and Ch. Pommerenke, On Bloch functions and normal functions, J. Reine Angew. Math. 270 (1974), 12-37.

3. S. Axler, Bergman spaces and their operators, Surveys of Some Recent Results in Operator Theory, Res. Notes in Math., Pitman, 1988, pp. 1-50.

4. R. Coifman, R. Rochberg, and G. Weiss, Factorization theorems for Hardy spaces in several variables, Ann. of Math. 103 (2) (1976), 611-635.

5. P. Duren, B. Romberg, and A. Shields, Linear functionals on $H^{p}$ spaces with $0<p<1, \mathrm{~J}$. Reine Angew. Math. 238 (1969), 32-60.

6. F. Forelli and W. Rudin, Projections on spaces of holomorphic functions in balls, Indiana Univ. Math. J. 24 (1974), 593-602.

7. C. Kolaski, A new look at a theorem of Forelli and Rudin, Indiana Univ. Math. J. 28 (1979), 495-499.

8. S. Krantz and D. Ma, Bloch functions on strongly pseudoconvex domains, Indiana Univ. Math. J. 37 (1988), 145-163. 
9. W. Rudin, Function theory in the unit ball of $\mathbb{C}^{n}$, Springer-Verlag, Berlin, Heidelberg, New York, 1980.

10. A. Shields and L. Williams, Bounded projections, duality, and multipliers in spaces of analytic functions, Trans. Amer. Math. Soc. 162 (1971), 287-302.

11. R. Timoney, Bloch functions in several variables I, Bull. London Math. Soc. 12 (1980), 241267.

12. __ Bloch functions in several variables II, J. Reine Angew. Math. 319 (1980), 1-22.

13. K. Zhu, Duality and Hankel operators on the Bergman spaces of bounded symmetric domains, J. Funct. Anal. 81 (1988), 260-278.

14. The Bergman spaces, the Bloch space, and Gleason's problem, Trans. Amer. Math. Soc. 309 (1988), 253-268.

Department of Mathematics, Michigan State University, East Lansing, Michigan 48824

Current address: Department of Applied Mathematics, Korea Advanced Institute of Science and Technology, P.O. Box 150, Cheong-Ryang, Seoul 130-650, Korea 